\title{
Dendritic cell entrapment within the pregnant uterus inhibits immune surveillance of the maternal/fetal interface in mice
}

\author{
Mary K. Collins, ${ }^{1}$ Chin-Siean Tay, ${ }^{1}$ and Adrian Erlebacher ${ }^{1,2}$ \\ 1Department of Pathology and 2NYU Cancer Institute, New York University School of Medicine, New York, New York, USA.
}

\begin{abstract}
Embryo implantation induces formation of the decidua, a stromal cell-derived structure that encases the fetus and placenta. Using the mouse as a model organism, we have found that this tissue reaction prevents DCs stationed at the maternal/fetal interface from migrating to the lymphatic vessels of the uterus and thus reaching the draining lymph nodes. Strikingly, decidual DCs remained immobile even after being stimulated with LPS and exhibiting responsiveness to CCL21, the chemokine that drives DC entry into lymphatic vessels. An analysis of maternal $\mathrm{T}$ cell reactivity toward a surrogate fetal/placental antigen furthermore revealed that regional $\mathrm{T}$ cell responses toward the fetus and placenta were driven by passive antigen transport and thus the tolerogenic mode of antigen presentation that predominates when there is negligible input from tissue-resident DCs. Indeed, the lack of involvement of tissue-resident DCs in the T cell response to the fetal allograft starkly contrasts with their prominent role in organ transplant rejection. Our results suggest that DC entrapment within the decidua minimizes immunogenic $T$ cell exposure to fetal/placental antigens and raise the possibility that impaired development or function of the human decidua, which unlike that of the mouse contains lymphatic vessels, might lead to pathological $\mathrm{T}$ cell activation during pregnancy.
\end{abstract}

\section{Introduction}

Tissue-resident DCs face two opposing challenges. On one hand, they must be alert to pathogens so they can prime $\mathrm{T}$ cells in the draining LNs. On the other hand, they must avoid activating $\mathrm{T}$ cells specific for innocuous antigens or else risk inducing autoimmune disease and pathogenic tissue destruction. An extreme example of this dichotomy comes from the case of pregnancy, where DCs normally charged with the task of protecting the uterus from infection must now accommodate the novel antigens expressed by the fetus and placenta. Indeed, inflammatory cytokine production and $T$ cell activation induced by DCs migrating from the pregnant uterus to the uterine LNs would be expected to have a major, deleterious effect on pregnancy outcome, even if this outcome were to fall short of overt fetal rejection. Several direct mechanisms of $\mathrm{T}$ cell suppression have therefore been evaluated in the context of pregnancy $(1,2)$; however, little consideration has been given to the possibility that differential DC behavior in the pregnant versus nonpregnant uterus might in itself be important for preventing anti-fetal/placental $T$ cell activation.

In a recent study based upon the transgenic expression of OVA as a surrogate fetal/placental antigen, we found that APCs exclusively of maternal origin mediated the proliferation and clonal deletion of naive anti-fetal/placental $\mathrm{T}$ cells (3). However, these $\mathrm{T}$ cells remained surprisingly ignorant of antigen over the first half of gestation, despite clear antigen expression by the developing placenta as early as E7.5, and only responded in a robust fashion when antigen started hematogenously shedding from the placenta on E10.5, approximately 6 days after implantation. It also appeared that shed antigen could travel in a non-cell-associated form via the regional lymphatics, since it started accumulating on nonmigra-

Conflict of interest: The authors have declared that no conflict of interest exists. Nonstandard abbreviations used: B6, C57BL/6; MHCII, MHC class II. Citation for this article: J. Clin. Invest. 119:2062-2073 (2009). doi:10.1172/JCI38714. tory follicular DCs (FDCs) in the uterine LNs earlier than in other LNs (3). These results suggested that $T$ cell recognition of the fetus and placenta was mediated in part by the passive transport of shed placental antigen and its local uptake by spleen- and LN-resident APCs, but left open the role of uterine DCs in the overall anti-fetal/ placental $\mathrm{T}$ cell response.

Embryo implantation induces a localized tissue reaction in the uterus that profoundly influences the differentiation and trafficking of several leukocyte subtypes $(2,4)$. This reaction, called decidualization, entails the proliferation and differentiation of the endometrial stromal cells adjacent to the implanted blastocyst and their formation of a large, highly ordered structure (the decidua) that encases the fetus and placenta (5). Recently, two groups used transgene-mediated inducible ablation of leukocytes expressing the surface marker CD11c, which include DCs, to show that these cells regulate key aspects of the decidual reaction itself $(6,7)$, and one of these groups further showed that DCs are not required to actively maintain fetomaternal tolerance (6). However, whether local processes in the pregnant uterus regulate the prototypical T cell-activating function of tissue-resident DCs remains unknown.

Here, we study the effect of decidualization on uterine DCs and directly assess their role in the regional anti-fetal/placental $\mathrm{T}$ cell response. Since the pregnant uterus contains a complex mixture of leukocytes that variably express DC markers such as CD11c $(6,8-10)$, our analysis first required visualizing DC emigration from the nonpregnant uterus in order to identify those cells with true LN-homing potential. Upon embryo implantation, some migratory DCs come to reside within the decidua but, remarkably, remain uninvolved in $\mathrm{T}$ cell recognition of the fetus and placenta. Instead, the cells remain trapped within the tissue even after being stimulated with LPS and showing appropriate cell-intrinsic migratory behavior. Together, these results suggest that the formation of the decidua creates an environment prohibitive for the migration of tissue-resident DCs and 
A

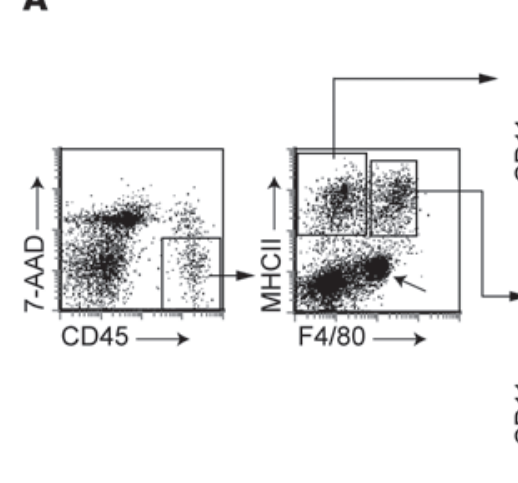

C

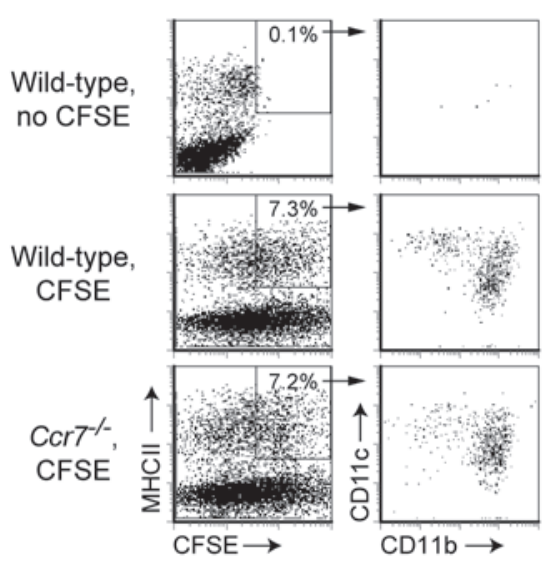

B

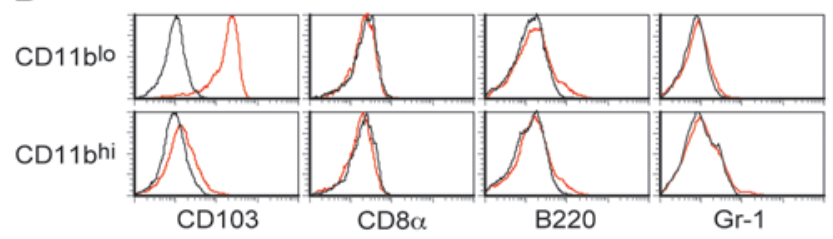

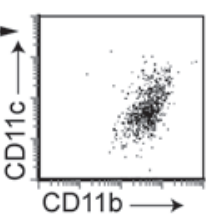

D
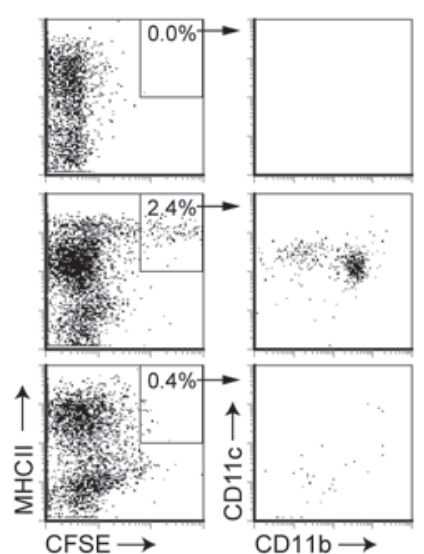

E
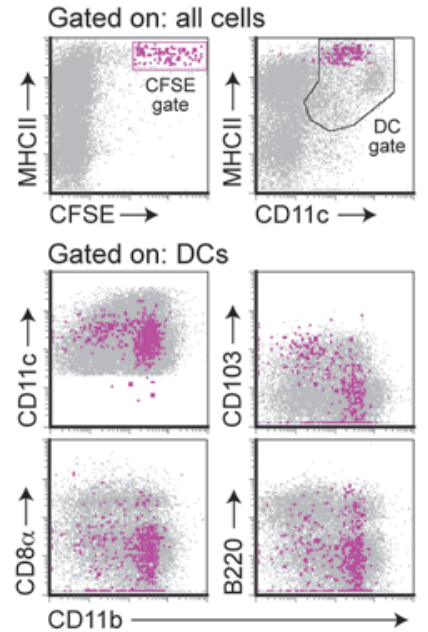

Figure 1

Identification of migratory DCs in the nonpregnant uterus. (A) A basic gating scheme to identify uterine DCs. Viable leukocytes were visualized using anti-CD45 antibodies in combination with the nucleic acid dye 7-aminoactinomycin D (7-AAD) to exclude dead cells. MHClI+F4/80- cells divided into 2 putative $D C$ subsets with $C D 11 \mathrm{C}^{+} \mathrm{CD} 11 \mathrm{~b}^{1 \mathrm{o}}$ and $\mathrm{CD} 11 \mathrm{C}^{+} \mathrm{CD} 11 \mathrm{~b}^{\text {hi }}$ surface phenotypes; $\mathrm{MHCll}+\mathrm{F} 4 / 80^{+}$cells were $\mathrm{CD} 11 \mathrm{c}^{\mathrm{l}}$. The diagonal arrow indicates an $\mathrm{MHCll}-\mathrm{F} 4 / 80^{+}$population largely consisting of eosinophils based upon their high side scatter characteristics (data not shown). (B) Cell-surface marker expression by MHCII+F4/80- DC subsets (red histograms). Black histograms show isotype control staining. (C and D) CCR7dependent arrival of CFSE + cells in the uterine LNs after CFSE labeling the uterus in situ. Flow cytometric analysis of total uterine cell suspensions (C) and respective uterine LN cells (D) 28 hours after transcervical CFSE injection. The plots are gated on alive $\mathrm{CD} 45^{+}$cells, and the percentage of $\mathrm{CD}_{4}{ }^{+} \mathrm{MHCll}^{+}$cells that are $\mathrm{CFSE}^{+}$is indicated. Data are representative of $n=4-6$ mice per group. (E) Cell-surface marker profiles of CFSE bright cells in the uterine LNs. CFSEbright gated cells (fuchsia) are show in the lower 4 panels overlaying the general distribution of DCs gated as indicated. In addition, CFSE bright cells did not express F4/80, Ly- $6 \mathrm{C}$, or Gr-1 (data not shown). In confirmation of the paraaortic and renal LNs being the draining LN of the murine uterus, only these LNs accumulated CFSE bright cells following transcervical CFSE injection into wild-type mice.

thus promotes immunological acceptance of the fetus by obviating the participation of these potent APCs in the regional antifetal/placental $\mathrm{T}$ cell response.

\section{Results}

Identification of migratory DCs in the nonpregnant uterus. We first used flow cytometry to characterize the uterine leukocytes of progesteronetreated virgin mice, using a high-dose hormonal regimen to simulate the environment of pregnancy and suppress the variation in leukocyte composition that occurs with the estrous cycle $(11,12)$. Putative DCs were identified in cell suspensions as constituting more than $90 \%$ of the MHC class II-positive $\left(\mathrm{MHCII}^{+}\right)$cells that were negative for the macrophage marker F4/80 (Figure 1A). These $\mathrm{MHCII}^{+} \mathrm{F} 4 / 80^{-}$ cells were already known to have high $\mathrm{T}$ cell immunostimulatory capacity compared with the $\mathrm{MHCII}+\mathrm{F} 4 / 80^{+}$cells (13), and we furthermore found that they divided into the $\mathrm{CD} 11 \mathrm{c}^{+} \mathrm{CD} 11 \mathrm{~b}^{\mathrm{lo}} \mathrm{CD} 103^{+}$and
CD11 ${ }^{+} \mathrm{CD} 11 \mathrm{~b}^{\text {hi }} \mathrm{CD} 103^{-}$DC subsets seen at other mucosal surfaces (14-17) (Figure 1, A and B). Neither subset expressed appreciable levels of CD8 $\alpha$ or B220, which are markers for lymphoid and plasmacytoid DCs, respectively, nor did the cells express the monocyte and granulocyte marker Gr-1 (Figure 1B).

To demonstrate that the $\mathrm{MHCII}^{+} \mathrm{F} 4 / 80^{-}$cells had LN-homing capacity, we visualized their appearance in the uterine LNs (the paraaortic and renal LNs in the mouse) after labeling the uterus in situ with the fluorescent dye CFSE. CFSE was injected into the uterine lumen via a nontraumatic transcervical approach that nonspecifically labeled all uterine cell types near the mucosal surface (Figure 1C and see below) and induced the appearance 28 hours later of a distinct $\mathrm{MHCII}^{+} \mathrm{CFSE}^{\text {bright }}$ cell population in the uterine LNs of wild-type C57BL/6 (B6) mice (Figure 1D). These cells subdivided into the same $C D 11 \mathrm{c}^{+} \mathrm{CD} 11 \mathrm{~b}^{\text {lo }}$ and $\mathrm{CD} 11 \mathrm{c}^{+} \mathrm{CD} 11 \mathrm{~b}^{\text {hi }}$ DC subsets present in the uterus and were collectively apparent 
A
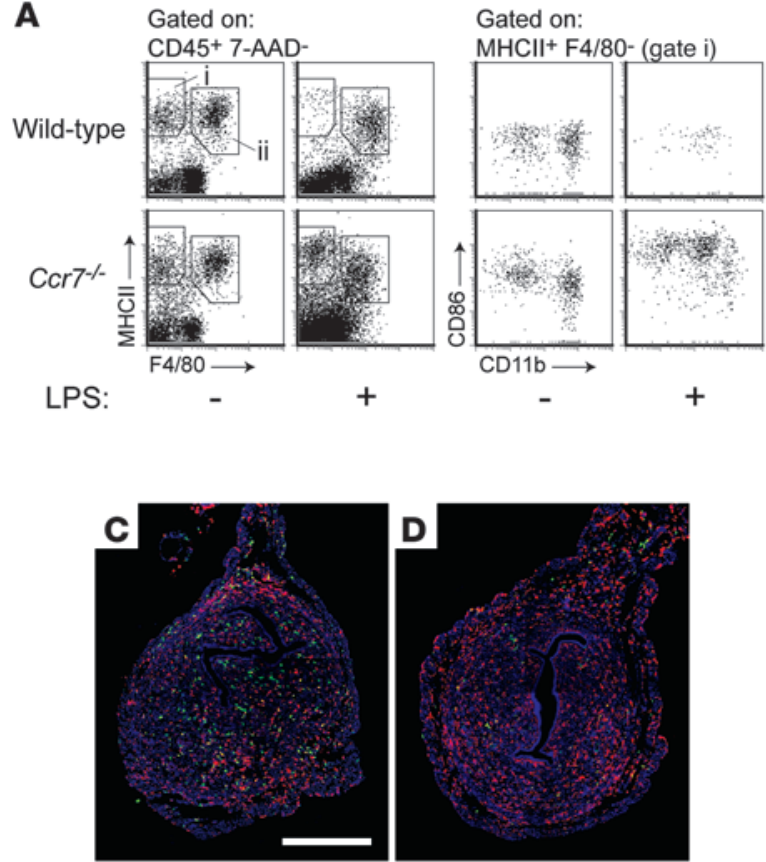

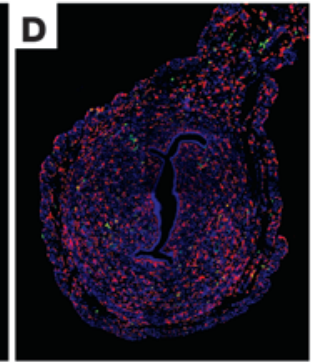

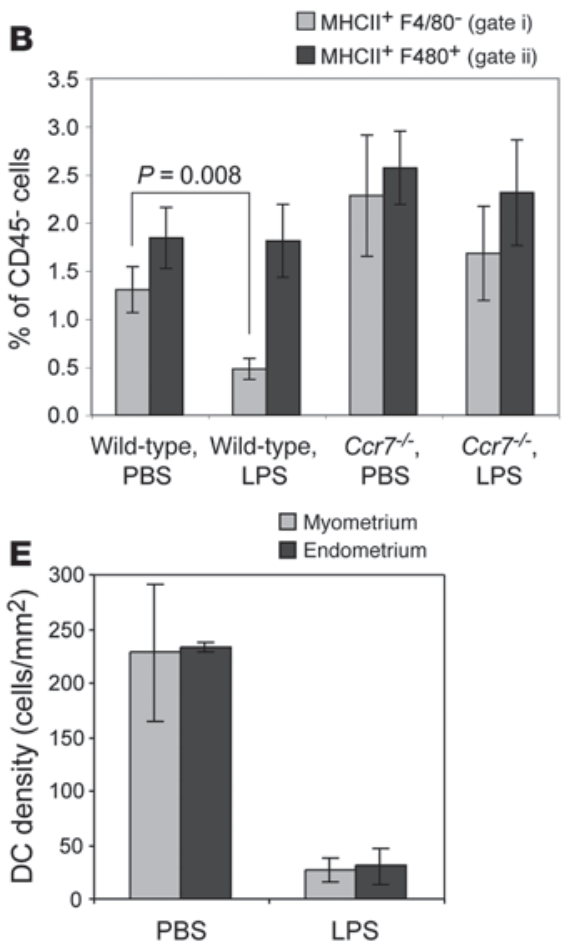

Figure 2

CCR7-dependent selective loss of DCs from the nonpregnant uterus following LPS stimulation. (A) Flow cytometric analysis of uterine leukocytes 28 hours after intravenous LPS injection. The number of cells shown for each mouse is normalized to a fixed number of CD45- non-rbc, which we used as estimates of uterine parenchymal cell number. CD86 expression levels by those few uterine DCs remaining in LPS-treated wild-type mice varied between individual mice. (B) Cell numbers for LPS- and control PBS-treated mice were calculated by flow cytometry using the gates shown in $\mathbf{A}$ and were normalized to CD45- non-rbc. Data show mean \pm SEM of $n=6-7$ mice per group, compiled from 3 independent experiments. (C and D) Representative sections of uteri from PBS- (C) and LPS-treated (D) mice, immunostained with anti-MHCII (green) and anti-F4/80 (red) antibodies. DCs are pure green ( $\left.\mathrm{MHCl}{ }^{+} \mathrm{F} 4 / 80^{-}\right)$cells. Color intensities in both images were subjected to the same set of nonlinear adjustments so that the cells would be visible at low magnification. Scale bar: $0.5 \mathrm{~mm}$. (E) Histomorphometric quantification of DC densities in the myometrium and endometrium of PBS- and LPS-treated mice (mean $\pm \mathrm{SD} ; n=3$ mice per group).

as MHCII ${ }^{\text {hi }} \mathrm{CD} 11 \mathrm{c}^{\mathrm{int}}$, as expected for migratory DCs (18) (Figure 1, $\mathrm{D}$ and $\mathrm{E})$. In contrast, uterine $\mathrm{LN}$ cells of $\mathrm{Ccr} 7^{-1-}$ mice, which lack the CCL21 receptor CCR7 and thus provide useful negative controls because of their profound defect in DC migration to the LNs (19-21), showed only a broad, low level of labeling, consistent with passive diffusion of the dye, and those few cells gated as CFSE ${ }^{\text {bright }}$ did not form discrete subpopulations (Figure 1D).

We also confirmed that the $\mathrm{MHCII}^{+} \mathrm{F} 4 / 80^{-}$cells were DCs by visualizing their LPS-induced maturation and CCR7-dependent emigration from the uterus. Intravenous LPS injection induced approximately $65 \%$ of these cells to disappear from the uteri of wild-type mice within 28 hours, with similar effect on the CD $11 b^{\text {lo }}$ and $C D 11 b^{\text {hi }}$ subsets. In contrast, the uteri of LPS-treated $\mathrm{Cr} 7^{-/-}$mice showed no significant loss of $\mathrm{MHCII}^{+} \mathrm{F} 4 / 80^{-}$cells, although the cells had increased expression of both MHCII and the maturation marker CD86 (Figure 2 , A and B). Consistent with their identity as mature macrophages, uterine $\mathrm{MHCII}^{+} \mathrm{F} 4 / 80^{+}$cells variably induced CD86 expression in response to LPS (data not shown) but did not significantly decrease in number in wild-type or $\mathrm{Ccr}^{-/-}$mice (Figure 2B). Importantly, double MHCII (green) and F4/80 (red) immunostaining of uterine cross sections revealed that $\mathrm{MHCII}^{+} \mathrm{F} 4 / 80^{-} \mathrm{DCs}$ (cells staining pure green) were present at equal densities in the endometrium and myometrium (Figure 2,
C and E) and were equivalently mobilized from these two tissue layers by LPS (Figure 2, D and E). These results demonstrated that DCs can exit both tissue layers of the nonpregnant uterus.

Tissue-resident DCs do not contribute to $T$ cell recognition of the fetus and placenta. Next, we used our recently developed mating system (3) to test whether uterine DCs participate in the maternal $\mathrm{T}$ cell response toward the fetus and placenta. This system involves mating female mice to Act-mOVA transgenic males (22), in order to express a transmembrane form of OVA in placental trophoblasts directly at the maternal/fetal interface, and then assessing the proliferative response of CFSE-labeled TCR transgenic anti-OVA $\mathrm{T}$ cells adoptively transferred at different stages of gestation. As mentioned above, $\mathrm{T}$ cells in this system showed only a minimal response over the first half of gestation. However, we also previously noted that once $\mathrm{T}$ cell proliferation began at mid-gestation, it was initially more robust in the uterine LNs than in nondraining LNs (e.g., the subcutaneous LNs) (3). Although this early, regional phase of $\mathrm{T}$ cell proliferation implicated OVA transport via the draining lymphatic vessels, it was unclear whether it was mediated by migratory DCs or by passive processes. Thus, we addressed the role of peripheral tissue DCs in T cell recognition of the fetus and placenta by asking whether Act-mOVA-mated $\mathrm{C} c 7^{-/-}$females, like control B6 females, initially showed more OVA-driven T cell proliferation in their uterine LNs than in their subcutaneous LNs. 

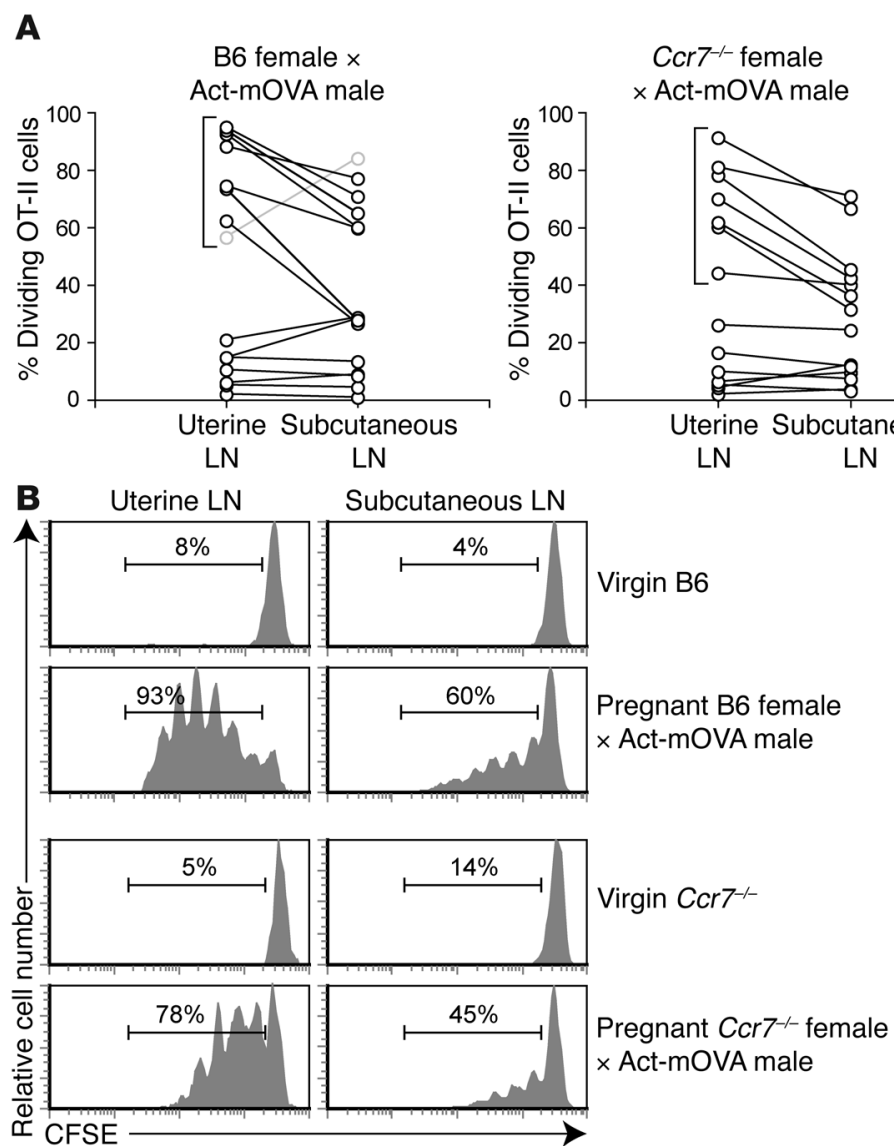
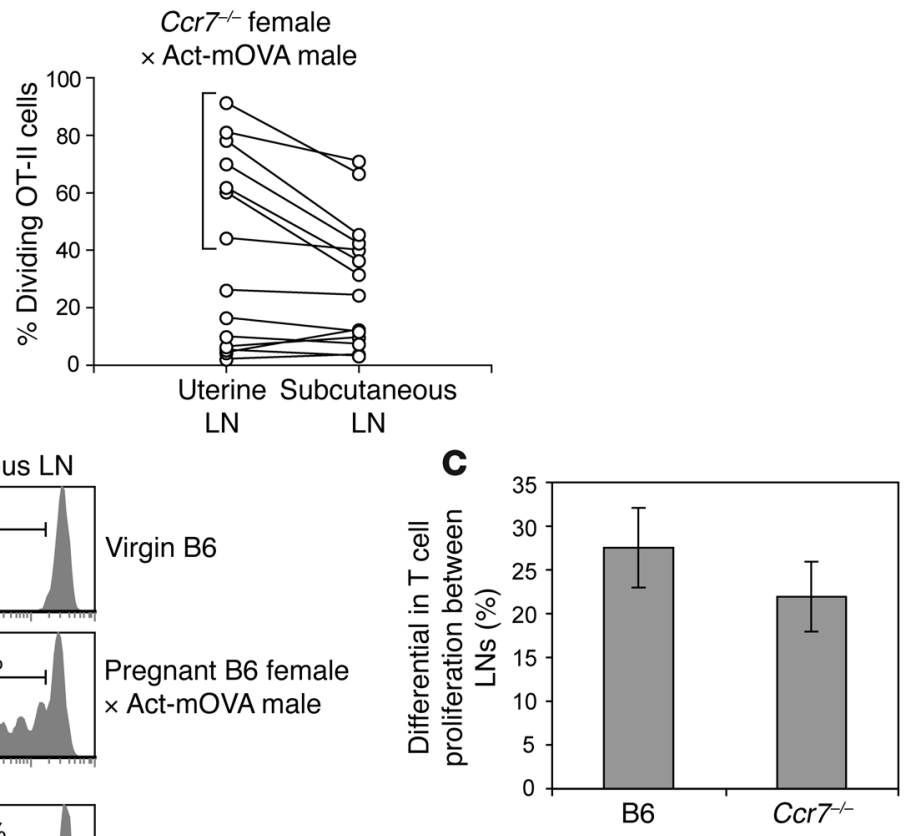

\section{Figure 3}

CCR7-independent presentation of a surrogate fetal/placental antigen in the uterine LN. (A) Quantification of OVA-driven OT-II cell proliferation in the uterine and subcutaneous LNs of Act-mOVA-mated wild-type B6 (left) and Ccr7-/- (right) mice. Mice were sacrificed 60 hours after being intravenously injected with $2 \times 10^{6}$ CFSE-labeled OT-II cells on a day of gestation ranging between E8.5 and 16.5. Individual mice are represented as linked data points. The percentage of dividing cells was determined as shown in B. Background proliferation in virgin and B6 $\times$ B6 pregnant mice was 4\%-14\%. (B) Representative OT-II CFSE dilution profiles for virgin B6, virgin Ccr7---, and the mice bracketed in A. The percentage of T cells that have undergone at least one division is indicated. (C) Average difference in percent proliferation \pm SEM between the uterine and subcutaneous LNs for the respective groups of mice bracketed in $\mathbf{A}$ ( $n=7$ mice per group). The B6 average excludes the atypical mouse represented by the gray symbols in $\mathbf{A}$.

In Figure 3, we display percentages of $\mathrm{CD} 4^{+} \mathrm{TCR}$ transgenic antiOVA OT-II T cells undergoing proliferation in the uterine versus subcutaneous LNs of individual mice as pairs of linked data points (Figure 3A), as well as CFSE dilution profiles representative of key groups of mice (Figure 3B). Each mouse was analyzed 60 hours after receiving an injection of CFSE-labeled OT-II cells on a gestation day ranging from E8.5 to E16.5. As expected from our previous study (3), we consistently observed more OT-II proliferation in the uterine LNs compared with subcutaneous LNs in those B6 females in which proliferation had begun yet was still submaximal (i.e., those mice indicated with brackets in Figure 3A, left panel; data for mice in which more than $95 \%$ of the OT-II cells in the uterine LNs were already proliferating were excluded from our analysis and, for clarity, results are not displayed). The corresponding set of $\mathrm{C} c r 7^{-/-}$females clearly showed a similar differential (Figure 3A, right panel), which on average was indistinguishable from that of wild-type $(P=0.37$, Figure 3C; these averages even exclude the data for the atypical mouse shown in gray in Figure $3 \mathrm{~A})$. Together, these data suggested that anti-fetal/placental $\mathrm{T}$ cell proliferation in the uterine LNs is driven not by DCs emigrating from the maternal/ fetal interface, but rather by the passive transport of antigen and its uptake by LN-resident DCs.

Decidualization induces a loss of DC tissue density. To understand the lack of involvement of uterine DCs in the anti-fetal/placental $\mathrm{T}$ cell response, we assessed their prevalence in the pregnant uterus and location relative to the fetus and placenta. Both DC subsets (Figure 4, gates $\mathrm{v}$ and vi, respectively) were apparent in the uterus from E4.5, which is approximately 12 hours after the initial attachment reaction between the blastocyst and the uterine epithelium (23), through E12.5. As the uterus grew during this period, DCs in the myometrium (Figure 4, lower left set of plots) increased in absolute number per implantation site, such that their tissue densities (cells per mg tissue) remained relatively constant (Figure 5A, left panels; see also Supplemental Figure 1; supplemental material available online with this article; doi:10.1172/JCI38714DS1). In contrast, DCs in the decidua (Figure 4, lower right set of plots) decreased in density approximately 6-fold from E4.5 to E12.5 while maintaining a relatively 

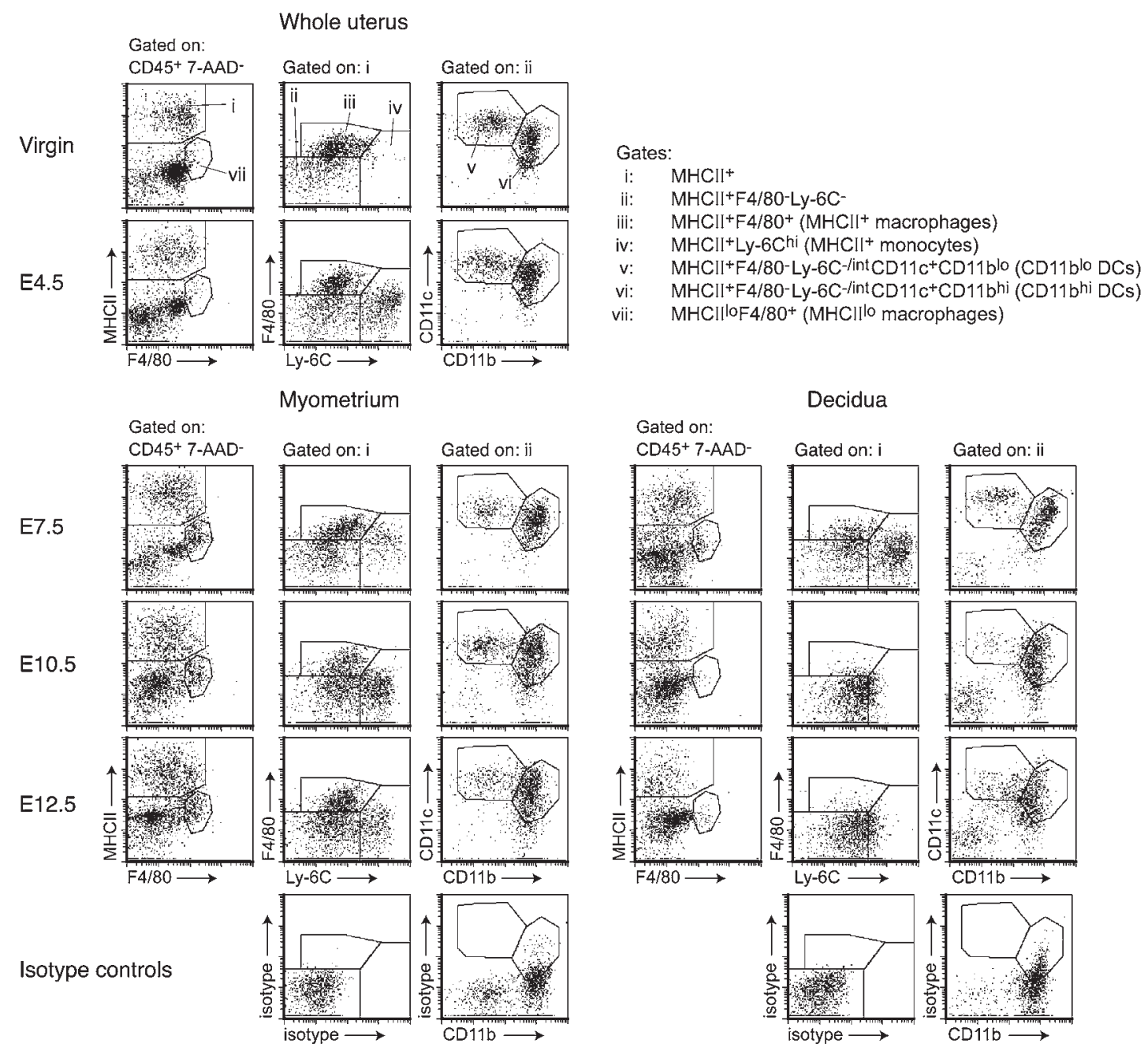

\section{Figure 4}

Identification of DCs in the pregnant uterus. Multicolor flow cytometry was used to characterize relevant cell populations based upon an initial CD45 ${ }^{+} 7-A A D^{-}$gate to identify viable leukocytes (not shown). Isotype control plots show data from E10.5 but are representative of all time points. Since the very little decidual tissue present on E4.5 cannot be easily dissected from the myometrium, data for this time point should be taken to represent the total cell composition of the pregnant uterus at this stage of gestation. In contrast to the uteri of progesterone-treated virgins, the E4.5 uterus contained a large number of $\mathrm{MHCll}+\mathrm{Ly}-6 \mathrm{C}^{\text {hi }}$ monocytes (gate iv) that were also apparent on E0.5 and in virgin females at a random stage of their estrous cycle (data not shown). These differences are consistent with the known effects of sex hormones on uterine leukocyte composition (13-15). The cells persisted in the myometrium through E12.5 and were present in the decidua through E7.5 as previously described (4). From E4.5 to E12.5, myeloid $\mathrm{MHCII}^{+}$cells in the decidua appeared increasingly homogeneous due to decreased F4/80 expression, increased basal Ly-6C expression, and the disappearance of the $\mathrm{MHCII+Ly}-6 \mathrm{C}^{\text {hi }}$ monocyte population by E10.5. The F4/80 staining is not as distinct as that shown in Figures 1 and 2 since our multicolor analysis required use of a dimmer fluorochrome.

constant number per implantation site (Figure 5A, left panels). Even when all myeloid $\mathrm{MHCII}^{+}$cells were taken as a single population of potential APCs, their tissue density was approximately $90 \%$ lower in the decidua compared with the myometrium by E10.5 (Figure 5A, right panels).

The decrease in decidual DC tissue density was confirmed by double anti-CD11c (red) and anti-MHCII (green) immunostaining of implantation sites (Figure 5B) and is all the more striking considering that $\mathrm{CD} 11 \mathrm{c}^{+} \mathrm{MHCII}{ }^{+}$cells would inclusively, but not exclusively, identify DCs due to the appreciable levels of CD11c expression on some $\mathrm{MHCII}^{+}$macrophages and monocytes (Supplemental Figure 2). These staining experiments furthermore revealed that decidual DCs were not directly apposed to the developing fetus and placenta and that the placenta itself contained no DCs on E10.5 and E14.5 (Figure 5B and Supplemental Figure 3).

Decidualization inbibits DC emigration to the draining LNs. Clearly, low DC densities in the decidua and placenta might limit the antifetal/placental T cell response. However, we were also struck by the observation that wild-type and $\mathrm{Ccr} 7^{-/-}$mice showed respectively similar DC tissue densities in the myometrium, decidua, and undecidualized endometrium, both when progesterone-treated virgins were compared and on E10.5 of pregnancy (Figure 5C). This not only argued against a major role for cell emigration in establishing uterine DC tissue densities in early gestation, but also implied minimal DC flux through the decidua at steady state. Interestingly, 
A
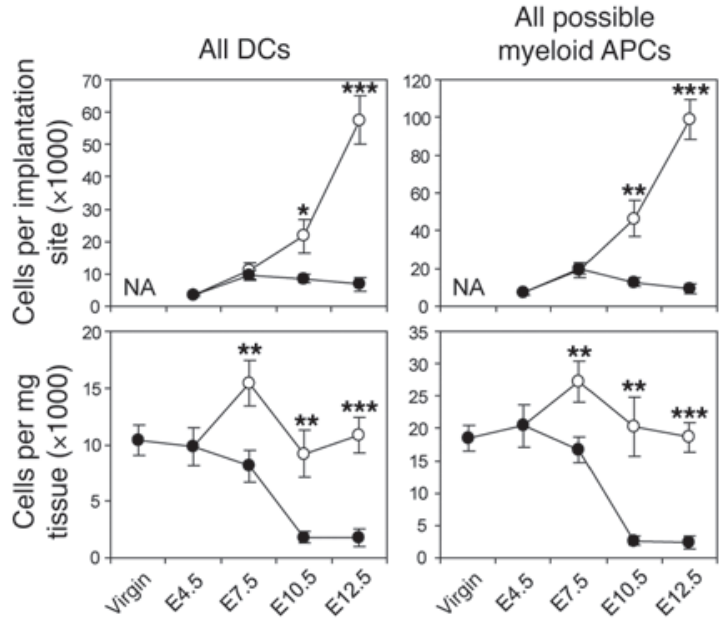

\section{B}
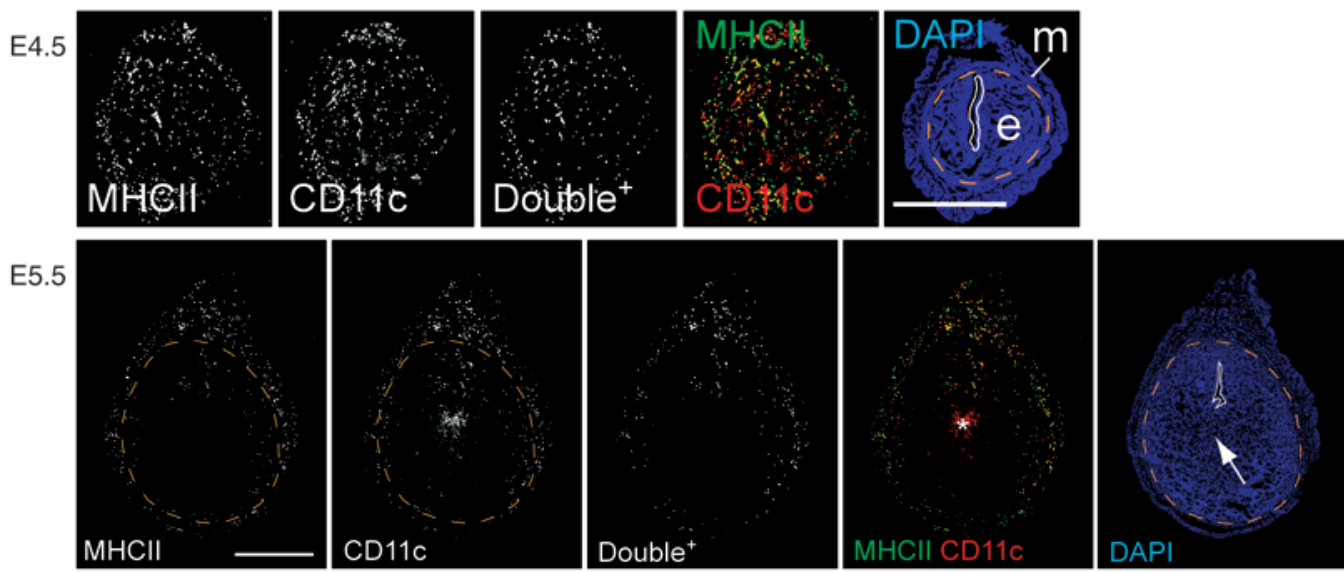

E7.5
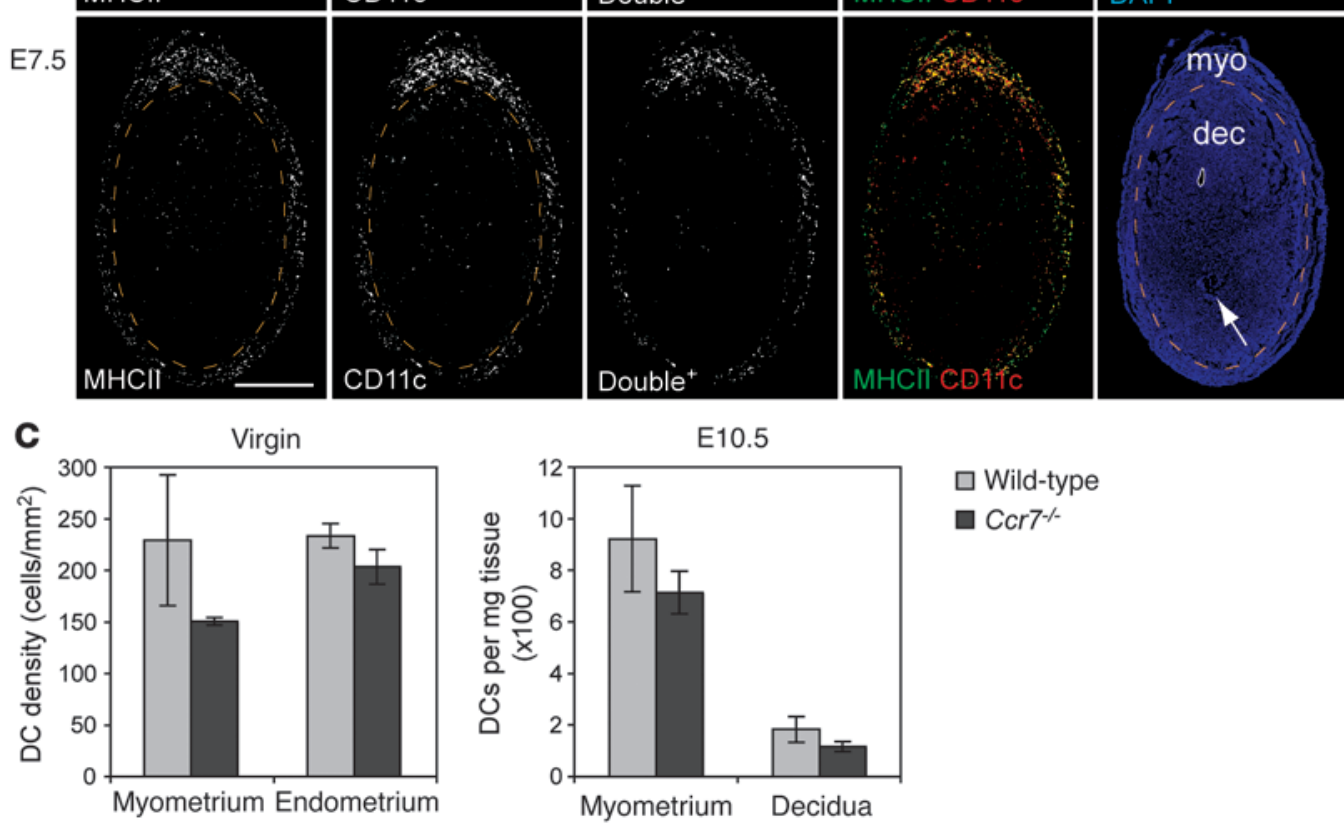

\section{Figure 5}

DC dynamics in the pregnant uterus. (A) Cell number per implantation site and cell number per milligram tissue (i.e., tissue density) for all DCs (sum of the $v$ and vi gates of Figure 4) and all possible myeloid APCs (sum of the iii, iv, v, and vi gates of Figure 4). Data show mean \pm SEM for $n=4-7$ mice per group. ${ }^{*} P<0.05$, ${ }^{* \star} P<0.02,{ }^{* \star *} P<0.005$ versus decidua. NA, not applicable. (B) Spatial distribution of uterine DCs during pregnancy. Transverse sections, with their mesometrial poles oriented upward, were double stained with antibodies against $\mathrm{MHCl}$ (green) and CD11c (red) and counterstained with DAPI. Single-channel images and combined MHCII/CD11C images after their "AND" gating (Double ${ }^{+}$cells; see Methods) are shown in black and white. Uterine lumens are outlined with solid white lines, and orange dashed lines demarcate the undecidualized endometrium (e) from the myometrium $(\mathrm{m})$ or the decidua (dec) from the myometrium (myo). The arrows indicate groups of trophoblasts lateral to implanted embryos. The asterisk indicates nonspecific staining. To visualize DCs in panoramic view, we manipulated the images as described in Methods; some are shown at higher magnification in Supplemental Figure 5. Scale bars: $1 \mathrm{~mm}$. (C) Uterine DC densities in wild-type versus $\mathrm{Ccr}^{-/-}$mice. Tissue densities in virgin mice, determined by histomorphometry, are shown as mean $\pm S D$ of $n=3$ mice per group; densities on E10.5, determined by flow cytometry, are shown as mean \pm SEM of $n=3-4$ mice per group. such low flux might be explained by the dramatic growth of the decidua in early pregnancy and the spatially restricted distribution of lymphatic vessels in the mouse uterus. Specifically, we noted that the confinement of LYVE-1+ lymphatic vessels to the myometrium
(24) positions them quite far away from the uterine lumen and maternal/fetal interface upon embryo implantation. We confirmed these data here (Figure 6, A, C, E, and G) and additionally found that the myometrial lymphatics were the only uterine structures to 

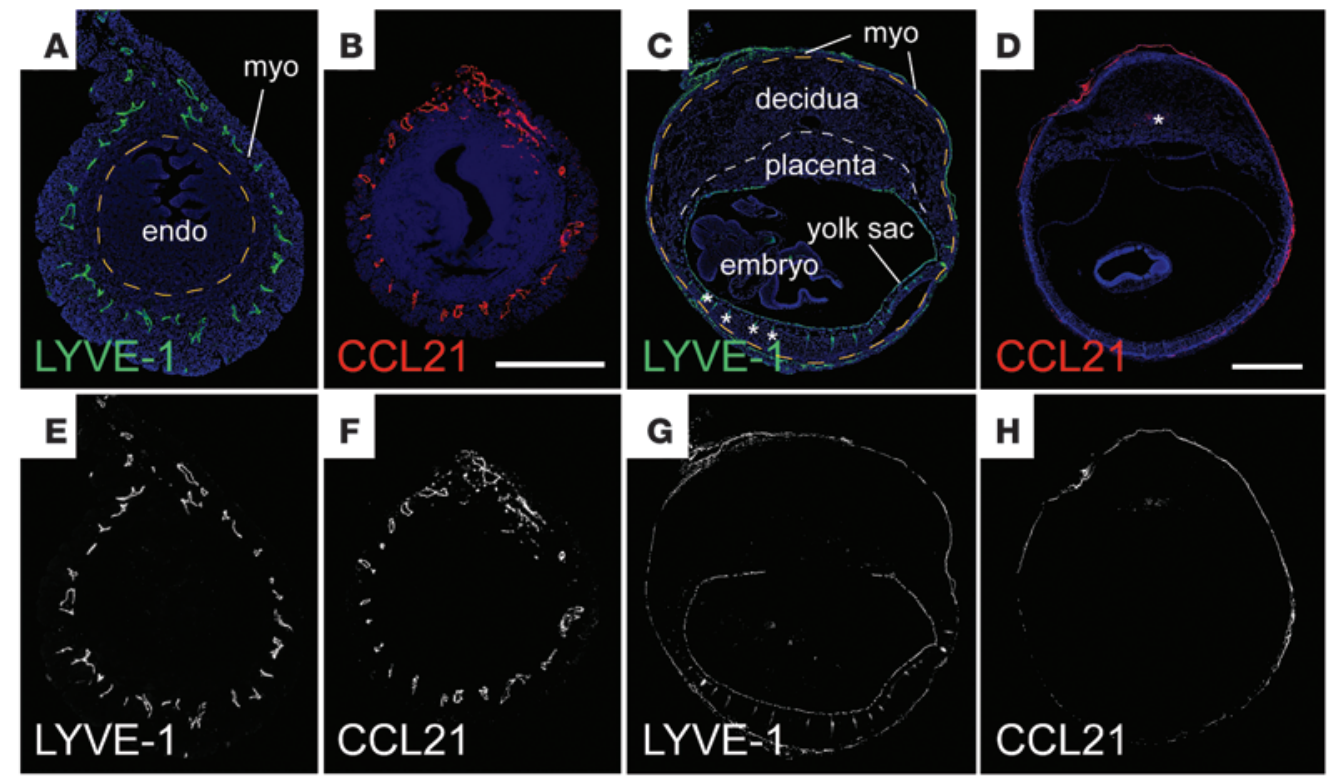

\section{Figure 6}

Lymphatic distribution and CCL21 expression in the nonpregnant and pregnant uterus. Nonpregnant uteri (A, B, E, and F; scale bar: $0.5 \mathrm{~mm})$ and E10.5 implantation sites $(\mathbf{C}, \mathbf{D}, \mathbf{G}$, and $\mathbf{H}$; scale bar: $1 \mathrm{~mm})$ were stained with antibodies against the lymphatic vessel marker LYVE-1 (A, C, $\mathbf{E}$, and $\mathbf{G}$ ) or CCL21 (B, D, F, and H) and counterstained with DAPI. Color images are shown in A-D; black and white images without the DAPI channel are shown in $\mathbf{E}-\mathbf{H}$ for clarity. The orange dashed lines in $\mathbf{A}$ and $\mathbf{C}$ demarcate the myometrium from endometrium and the myometrium from decidua, respectively. The white dashed line in $\mathbf{C}$ demarcates the placenta from decidua. In addition to lymphatic vessels, LYVE-1 is also expressed by yolk sac endothelial cells. The asterisks in $\mathbf{C}$ indicate some staining artifacts caused by tissue folding; the asterisk in $\mathbf{D}$ indicates some nonspecific staining also seen in the absence of primary antibody. Staining intensities were subjected to nonlinear adjustments so that structures would be visible at low magnification.

express PROX1, a transcription factor that specifies lymphatic vascular endothelial cell identity (Supplemental Figure 4). Even more importantly, these structures were the only ones to express CCL21 (Figure 6, B, D, F, and H). Indeed, transcript levels of Ccl21 were low in the decidua and high in the myometrium (and high in the subcutaneous LNs; Supplemental Figure 4), while transcript levels of Ccl19, which encodes the only other known ligand for CCR7, were low in both the decidua and myometrium (yet high in the subcutaneous LNs; Supplemental Figure 4). Other chemokines implicated in DC migration within peripheral tissues were expressed either at virtually undetectable levels in implantation sites (Ccl1, Ccl7, Ccl8, and $C c 120$ ) or at levels in the myometrium that were similar to or greater than those observed in the decidua (Cxcl12, Ccl2, Ccl5, and Ccl9). Together, these data suggested that DCs at the maternal/fetal interface would first have to migrate to the myometrium in order to reach the draining LNs. We therefore suspected that impediments to this initial step in DC migration might limit the participation of decidual DCs in the anti-fetal/placental T cell response.

To test this idea directly, we first asked whether CFSE ${ }^{+}$DCs would appear in the uterine LNs following CFSE injection into the decidual lumen. These experiments took advantage of a wellestablished technique to generate artificial decidua that involves injecting sesame seed oil into the uterine lumen of female mice rendered hormonally pseudopregnant and competent to decidualize by virtue of their copulation with vasectomized males. Artificial decidual tissue is similar in histological appearance and development to real decidual tissue (25) but is frequently induced over the entire length of both uterine horns. Thus, we could insure that contact between the CFSE and decidual tissue would be uninter- rupted by the stretches of undecidualized uterus present between real implantation sites and unimpeded by implanted embryos. Both oil and CFSE were injected via the transcervical approach in order to avoid surgical trauma to the uterus; CFSE was injected 3 days after oil injection, when the artificial decidua corresponded to that seen on E6.5 of pregnancy. Control undecidualized uteri were generated by injection of oil into females that remained unmated yet were given daily progesterone injections to simulate the hormonal environment of pseudopregnancy.

As shown in Figure 7B, transcervical CFSE injection labeled the subluminal cells of the decidua to a similar if not greater extent than it labeled the epithelial and subepithelial stromal cells of the undecidualized uterus (Figure 7B, inset), and in both cases labeling was apparent throughout the length of both uterine horns (data not shown). Strikingly, however, only approximately $4 \%$ of the MHCII ${ }^{\text {hi }}$ CD $11 c^{\text {int }}$ migratory DCs in LNs draining decidualized uteri became $\mathrm{CFSE}^{+} 28$ hours after injection, and those positive cells showed relatively dim CFSE fluorescence (Figure 7, C and D). In contrast, approximately $25 \%$ of the migratory DCs in LN draining undecidualized uteri became $\mathrm{CFSE}^{+}$, and many of these cells were CFSE $E^{\text {bright }}$, suggesting true DC migration. Indeed, transcervical CFSE injection into the decidualized uterus failed to give rise to any discrete $\mathrm{CFSE}^{\text {bright }}$ population in the draining LNs (or spleen), suggesting that no other decidual cell type could assume migratory DC-like behavior (data not shown). In contrast to our results with transcervical CFSE injection, many CFSE ${ }^{\text {bright }}$ DCs appeared in the uterine LNs after CFSE was painted onto the myometrial surface of the decidualized uterus, indicating that impaired DC migration from the decidua was specific for this tissue layer (data not shown). 


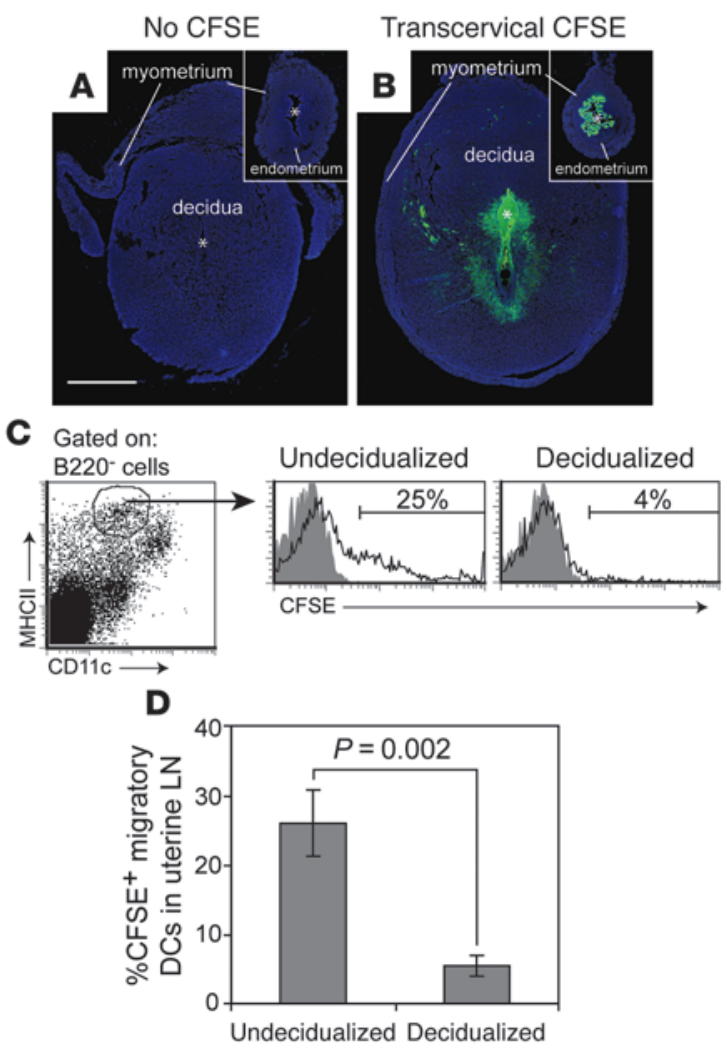

The above results showed that DC trafficking from the decidua to the LNs was minimal under noninflammatory, steady-state conditions. Remarkably, DCs also remained within the decidua after exposure to LPS, a powerful immune adjuvant that can induce DC emigration from a variety of tissues (26). Thus, DC numbers per implantation site and DC tissue densities in the decidua remained unchanged 28 hours after intravenous LPS injection on E6.5, whereas both parameters decreased approximately $85 \%$ in the myometrium (Figure 8A). By immunohistochemistry, selective DC loss from the myometrium was apparent as an equilibration of $\mathrm{MHCII}^{+} \mathrm{CD} 11 \mathrm{c}^{+}$cell densities between the myometrium and decidua (Figure 8, D and $\mathrm{E}$ ) that contrasted with the relatively high density of myometrial $\mathrm{MHCII}^{+} \mathrm{CD} 11 \mathrm{c}^{+}$cells in untreated mice (Figure 8, B and C).

Decidual DCs intrinsically retain maturation and migratory capacity but are trapped within the tissue. LPS-exposed DCs might remain within the decidua because they have undergone some cell-intrinsic change that either precludes their migration or renders them LPS nonresponsive. Alternatively, the cells might appear paralyzed because their environment is nonpermissive for cell migration. To evaluate these possibilities, we assessed the maturation status of uterine DCs in situ and their chemotactic response in vitro. First, we noted that CD86 and CCR7 expression levels were similarly elevated on DCs in both the E7.5 myometrium and decidua 12-15 hours after LPS injection on E6.5 (at which time myometrial DCs had not yet left the tissue) (Figure 9A) and that these DCs showed a similar degree of migration toward CCL21 when $\mathrm{MHCII}^{+}$cells from the two tissue layers were independently purified and subjected to Transwell chemotaxis assays immediately ex vivo (Figure 9B, left panel). In contrast, the $\mathrm{F} 4 / 80^{+}$fraction of the assayed cells remained immobile, as expected. These

\section{Figure 7}

Decidualization reduces DC migration from the uterus to the draining LNs. Mice with artificially decidualized or undecidualized uteri were injected transcervically with CFSE and sacrificed 28 hours later. (A and B) Transverse sections of uteri from uninjected (A) or CFSE-injected (B) mice were stained for CFSE using anti-FITC antibodies and counterstained with DAPI. Insets show undecidualized uteri magnified to the same extent as decidualized uteri. The myometrium has partially peeled away from the decidua in A. All images were subjected to same nonlinear adjustment so that CFSE staining intensities would be comparable, yet visible in a panoramic view. Scale bar: $1 \mathrm{~mm}$ (applies to all images). (C) Gating scheme and representative histograms showing the appearance of $\mathrm{CFSE}^{+}$migratory DCs in the uterine LNs. Cells were gated on B220- cells to exclude B cells and thus help clarify the $\mathrm{MHClI}{ }^{\text {hi CD11 }} \mathrm{C}^{\text {int }}$ migratory DC population (see Figure 1E). Shaded and open histograms are from untreated and CFSE-injected mice, respectively. The percentage of CFSE+ cells was determined by setting the lower bound of the marker to give approximately $1 \%$ positive cells in the non-CFSE-injected group. (D) Mean percentage of CFSE ${ }^{+}$migratory DCs ( \pm SEM; $n=6$ mice per group).

results thus suggested that decidual DCs retain LPS responsiveness and the same intrinsic ability to migrate toward CCL21 upon maturation as myometrial DCs.

Consistent with our in vivo data, however, decidual DCs failed to emigrate out of the tissue when E7.5 explants were cultured for 48 hours in medium containing CCL21 (Figure 9B, middle panel). This phenomenon appeared to require that the decidua be maintained as an intact tissue, as CCL21 attracted decidual DCs when we assayed total disaggregated decidual cells from LPStreated mice (Figure 9B, right panel); indeed, DCs within total disaggregated decidual cells were attracted to CCL21 to a similar extent as DCs within purified $\mathrm{MHCII}^{+}$cells (Figure 9B, compare left and right panels). In contrast, DC emigration out of myometrial explants (which on E7.5 contained the same number of DCs per implantation site as the decidua; Figure 5A) was induced by CCL21 over a detectable baseline level in the absence of chemokine (Figure 9B, middle panel), but total disaggregated myometrial cells consistently showed much less DC migration toward CCL21 than purified $\mathrm{MHCII}^{+}$myometrial cells (Figure 9B, left and right panels). While this latter result paradoxically suggest that factors in the myometrium, but not the decidua, actively retain DCs to some extent when placed in competition with exogenous CCL21, it is consistent with the higher myometrial expression of CCL21 itself and other chemokines (see Supplemental Figure 4), as well as the possibility that the myometrium might be generally more adhesive for DCs than the decidua. Indeed, these data collectively not only provide additional evidence of DC entrapment within the decidua, but also suggest that the decidua, when structurally intact, is unsupportive of chemokine-directed interstitial DC migration but does not actively retain the cells within the tissue.

\section{Discussion}

In this study, we present evidence that the decidua inhibits DC surveillance of the maternal/fetal interface and thus plays a pivotal role in preventing immune rejection of the fetus. This evidence is based on the identification of LN-homing uterine DCs as cells with $\mathrm{CD} 11 \mathrm{c}^{\text {hi }} \mathrm{MHCII}{ }^{+} \mathrm{F} 4 / 80^{-} \mathrm{CD} 11 \mathrm{~b}^{\text {lo }} \mathrm{CD} 103^{+}$and CD $11 \mathrm{c}^{\text {hi }} \mathrm{MHCII}^{+} \mathrm{F} 4 / 80^{-} \mathrm{CD} 11 \mathrm{~b}^{\text {hi }} \mathrm{CD} 103^{-}$surface phenotypes. A recent report also detected these populations in the E8.5 pregnant uterus but did not separately visualize them in the myometrium 

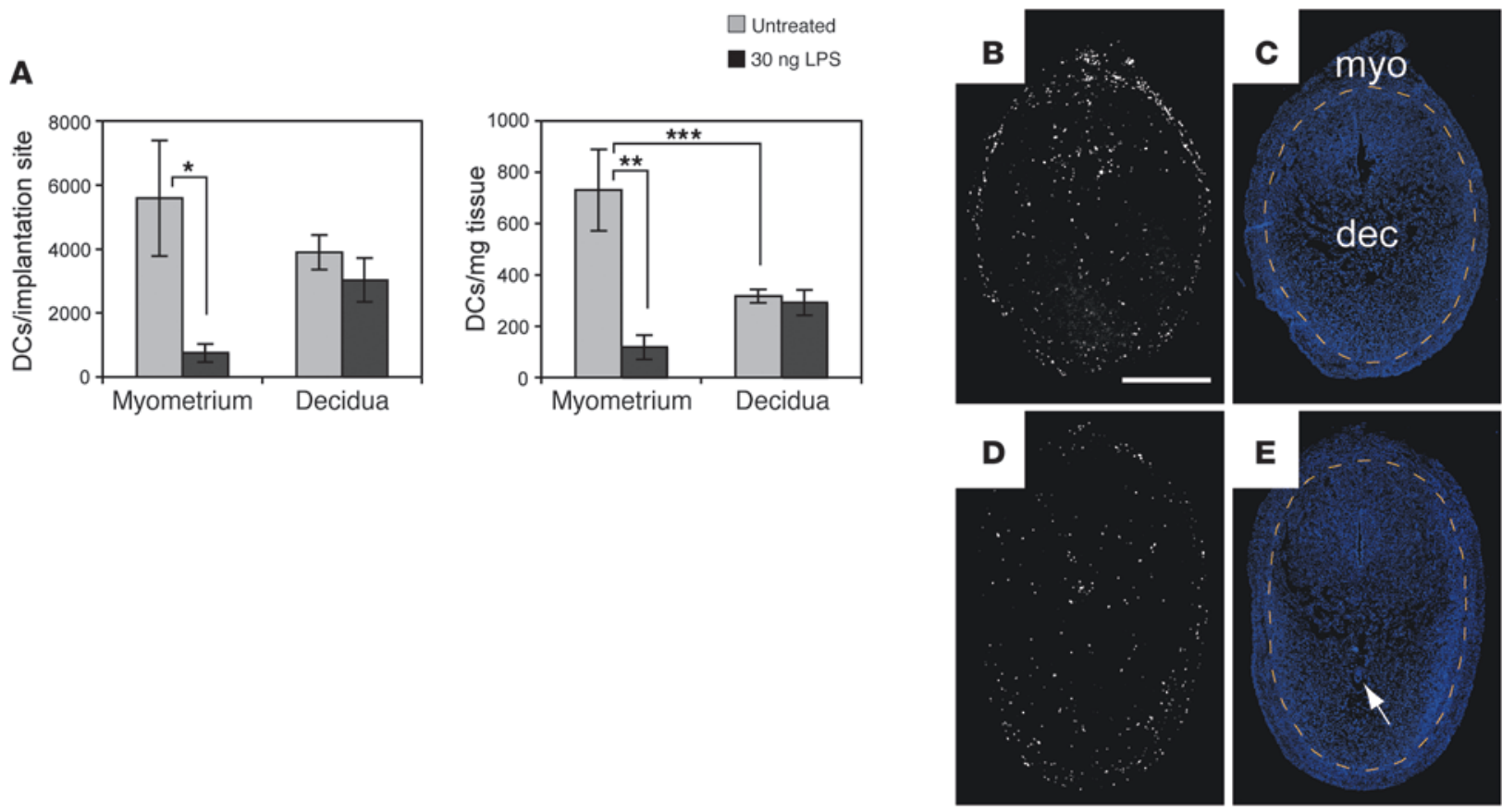

\section{Figure 8}

LPS fails to induce DC emigration from the decidua. E6.5 pregnant mice remained untreated or were injected intravenously with 30 ng LPS. (A) Flow cytometry-based quantification of DC cell numbers per implantation site and DC tissue densities 28 hours after injection. DCs were enumerated as MHClI+F4/80-CD11 chi cells. The data show mean \pm SEM for $n=6$ mice per group. ${ }^{\star} P=0.02,{ }^{* \star} P=0.004,{ }^{* \star *} P=0.03$. We obtained the same results when we considered each DC subset individually (data not shown). (B-E) Spatial distribution of DCs in implantation sites of untreated (B and $\mathbf{C}$ ) and LPS-treated (D and $\mathbf{E})$ mice 28 hours after injection. Transverse sections were double stained with anti-MHCII and anti-CD11c antibodies. (B and D) Combined images after their "AND" gating (i.e., Double+ cells as in Figure 5). (C and D) DAPI counterstaining. Orange dashed lines demarcate the decidua from the myometrium. The arrow indicates an implanted embryo. To visualize DCs in panoramic view, the images were manipulated as described in Methods. Scale bar: $1 \mathrm{~mm}$. Data are representative of $n=3-5 \mathrm{mice}$ per group.

versus decidua nor assess their migratory potential (10). Interestingly, another report that demonstrated a requirement for uterine

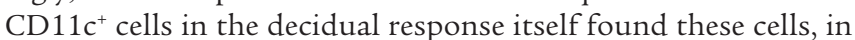
aggregate, to be largely $\mathrm{F} 4 / 80^{+}(6)$. While we cannot rule out the possibility that such cells have local DC-like function, our Transwell migration assay data and analysis of DC behavior in the nonpregnant uterus argue against $\mathrm{MHCII}{ }^{+} \mathrm{F} 4 / 80^{+}$cells having $\mathrm{LN}$ homing potential. Strikingly, the decidua progressively loses DC tissue density over the first half of postimplantation development (E4.5-E12.5) and appears to possess only those DCs already present in the endometrium at the time of implantation. In contrast, DC tissue densities in the growing myometrium remain relatively constant. Provocatively, the distribution of DCs in the myometrium and decidua parallel that previously described for macrophages (27-29) (see Supplemental Figures 1 and 3), suggesting that the tissue layer-specific densities of both cell types are differentially regulated by the same cellular and molecular pathways.

Although the contribution of decidual DCs to the anti-fetal/placental $\mathrm{T}$ cell response might be limited by their low tissue density, it is likely more critical that these cells are unable to exit the tissue and migrate to the uterine LNs. The entrapment of DCs within the decidua is supported by 4 independent lines of investigation. First, in contrast to the large number of $\mathrm{CFSE}^{+} \mathrm{DCs}$ that appeared in the uterine LNs after we labeled the undecidualized uterus with CFSE, only few such cells appeared after CFSE labeling of the decidua. Second, LPS induced the disappearance of DCs from the myome- trium but not the decidua, while similar experiments performed on nonpregnant mice showed equal loss of DCs from the myometrium and endometrium in a CCR7-dependent fashion. Third, DCs failed to emigrate from the decidua when explanted tissue was placed in CCL21-containing medium. Last, wild-type and $\mathrm{C} c 7^{-1-}$ female mice showed an equivalent early phase of fetal/placental antigen presentation in the uterine LNs, indicating that the regional $\mathrm{T}$ cell response to the fetus and placenta is not driven by migratory DCs. Bolstering this direct evidence is our data on $\mathrm{Cr}^{-1-}$ mice suggesting that the steady-state flux of DCs through the decidua is quite low.

A failure of maternal DCs to monitor the maternal/fetal interface has major implications for afferent mechanisms of fetomaternal tolerance. By analogy to solid organ transplantation (30), two distinct cellular pathways involving migratory DCs would be expected to prime anti-fetal/placental T cells in the uterine LNs. We previously showed that the "direct" pathway is inoperative over the entirety of gestation, most likely because the fetus simply does not contain a population of migratory DCs analogous to the donor DCs that emigrate from solid organ transplants (3). Here, we show that the "indirect" pathway, which would be driven by maternal DCs originating from the maternal/fetal interface, is largely, if not entirely, attenuated over the first half of gestation (through about E12.5). The maternal $\mathrm{T}$ cell response to the implanted embryo thus appears akin to what might transpire if an organ depleted of "passenger leukocytes" were to be transplanted into an anatomical location rendered devoid of a draining lymphatic vasculature, two 
A

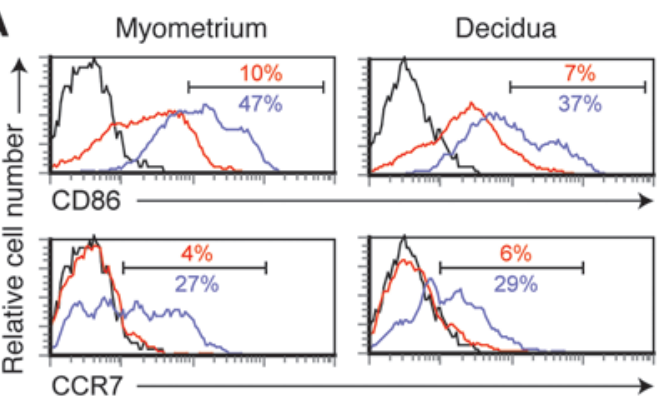

B

$\square \mathrm{DCs}$; media alone $\quad \square \mathrm{MHCl}+$ macrophages; media alone

aCs; media + CCL21 $\square \mathrm{MHCI}+$ macrophages; media + CCL21
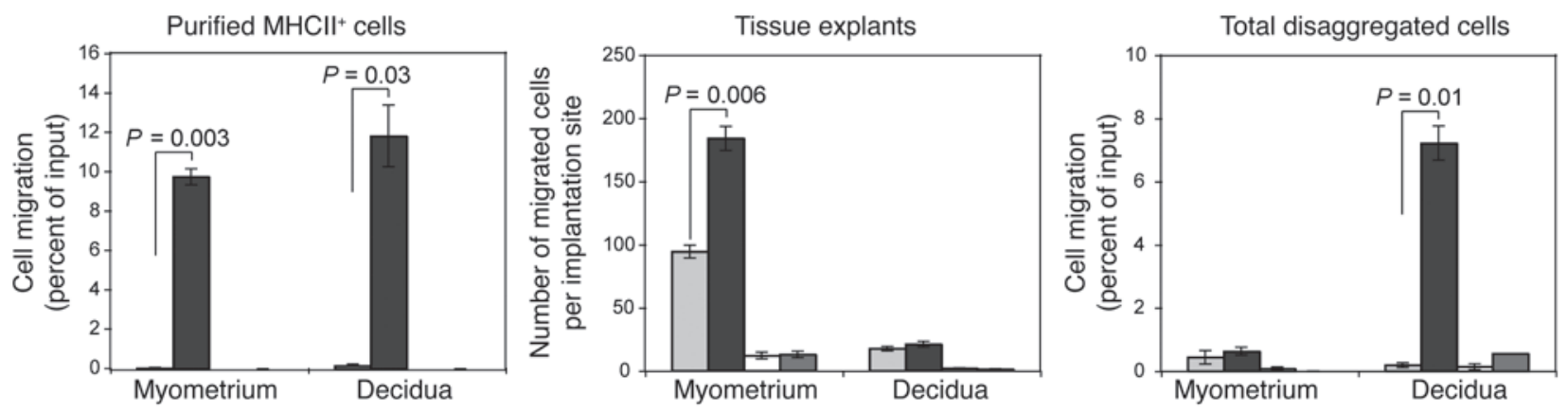

Figure 9

Decidual DCs retain their cell-intrinsic maturation and migratory capacity yet are trapped within the tissue. (A) CD86 and CCR7 expression levels on $\mathrm{MHCll}+\mathrm{F} 4 / 80^{-} \mathrm{CD} 11 \mathrm{c}^{\text {hi }}$ uterine DCs. The cells were harvested on E7.5 from untreated mice or mice that had been intravenously injected 15 hours earlier with $30 \mathrm{ng}$ LPS. Percentages of cells with the indicated levels of expression are shown. Data are representative of $n=3$ mice per group. (B) Ex vivo migration of cells from the myometrium or decidua toward medium alone or medium containing CCL21. The Transwell migration of DCs $\left(\mathrm{MHClI}+\mathrm{F} 4 / 80^{-} \mathrm{CD} 11 \mathrm{c}^{\text {hi }}\right)$ and $\mathrm{MHCll}^{+}$macrophages $\left(\mathrm{MHCll}+\mathrm{F} 4 / 80^{+}\right)$from $\mathrm{MHCll}^{+} \mathrm{MACS}$-purified cells (left panel) or total disaggregated cells (right panel) are expressed as a percentage of their respective input cell number. The cells were isolated on E7.5 fifteen hours after the intravenous injection of $30 \mathrm{ng}$ LPS. Data are mean \pm SEM of 2 wells per group. These experiments were replicated 3 times with similar results. The middle panel shows the total number of cells (mean \pm SEM of 5 mice per group compiled over 3 independent experiments) that emigrated out of intact tissue explants.

experimental manipulations that dramatically and independently prolong graft survival $(31,32)$. Indeed, DC entrapment within the decidua explains our inability to detect $\mathrm{T}$ cell responses to fetal/ placental OVA early in gestation. Although we cannot rule out the possibility that DCs exit the decidua later in pregnancy, this would seem unlikely given our current set of data.

In addition to DCs, our CFSE tracking experiments suggested that no other cell type could migrate in appreciable numbers from the decidua to the spleen or LNs. Thus, the response of naive maternal $\mathrm{T}$ cells to natural fetal/placental antigens is likely mediated through the cell-free, passive transport of these antigens via the blood or regional lymphatics and their local uptake by spleen- and LN-resident DCs. This pathway is clearly apparent with our surrogate antigen, OVA, which is shed from the placenta in immunologically detectable quantities starting at mid-gestation. Indeed, shed OVA also accumulates systemically on FDCs, a nonmigratory cell type resident within secondary lymphoid organs that binds complement-fixed, cell-free material (3). Although the specific mechanism has remained elusive, $\mathrm{T}$ cell priming is impaired when peripheral tissue antigens arrive in the LN solely by passive transport and are thus presented exclusively by LN-resident DCs $(33,34)$. Not only might this explain why anti-OVA $\mathrm{CD}^{+} \mathrm{T}$ cells are not primed to fetal/placental OVA despite their proliferative response (3), but it also predicts that the presentation of natural fetal/placental antigens would be similarly tolerogenic.
Strikingly, decidual DCs retain maturation capacity in situ and the intrinsic ability to become CCL21-responsive upon stimulation. On the other hand, our Transwell migration assays suggest that they are not actively retained within the tissue, a finding that is consistent with our chemokine expression data indicating that only the myometrium produces DC-attracting chemokines. Thus, DC entrapment within the decidua is likely the result of downstream, DC-extrinsic factors that more passively preclude DC emigration. One possibility is that the specialized extracellular matrix produced by decidual stromal cells provides an insufficient scaffold for interstitial DC migration. Along with many unusual features $(35,36)$, this matrix contains low amounts of hyaluronan, a molecule known to be required for DC migration likely through interactions with its cell-surface receptor CD44 (37-41). Second, it is possible that the periphery of the decidua creates some sort of physical barrier, although the relatively homogenous distribution of decidual DCs within LPS-treated mice (Figure 8D) would argue against this idea. Last, decidualization might disrupt the formation of chemokine gradients or simply increase the distance between the myometrium and decidual DCs to such an extent that they can no longer sense CCL21 produced by myometrial lymphatic vessels.

Interestingly, the human endometrium, like the mouse endometrium, is largely devoid of lymphatic vessels, whereas the human decidua shows robust lymphangiogenesis unlike that in the mouse $(24,42)$. Thus, an outstanding question is whether human 
decidual DCs are able to exit the uterus and, if so, whether their stimulation of anti-fetal/placental $\mathrm{T}$ cell responses in some circumstances might contribute to poor pregnancy outcomes. Along the same lines, a pathological decidual response in itself might promote inappropriate or overly robust $\mathrm{T}$ cell activation mediated by migratory DCs. Interestingly, our demonstration that uterine DC migration can be inhibited by decidualization, a stromal cell developmental process, suggests that analogous processes might also inhibit the sentinel function of other tissue-resident DCs. For example, tumor-associated stromal cells might inhibit the immune surveillance of tumor antigens because they produce an extracellular matrix that cannot support interstitial DC migration or because their proliferation sufficiently deranges tissue architecture so as to interfere with the function of lymphatic vessel-derived CCL21. Similar stromal cell-based processes might be of clinical use to prevent the rejection of transplanted tissues and cells.

\section{Methods}

Mice. B6CBAF1, B6, and Act-mOVA mice (22) were obtained from The Jackson Laboratory. C $c r 7^{-1-}$ mice (20) were a gift from R. Förster (Hannover Medical School, Hannover, Germany) and M. Lipp (Max-Delbrück-Center for Molecular Medicine, Berlin, Germany) and had been backcrossed onto a B6 background $\left(\mathrm{N}_{10}\right)$ by S. Lira (Mount Sinai School of Medicine, New York, New York, USA). Vasectomized ICR males and OT-II Rag1 $1^{-/-}$mice were obtained from Taconic. Females were mated to either B6 males to induce pregnancy or vasectomized ICR males to induce pseudopregnancy. Noon of day of the copulation plug was designated E0.5. Mice were maintained in a specific pathogen-free facility, and all experiments were approved by the New York University School of Medicine Institutional Animal Care and Use Committee. Genotyping of Act-mOVA embryos was performed as previously described (3).

Transcervical injection. Mice were anesthetized with a ketamine/xylazine/ acetopromazine cocktail. As previously described (43), the cervix was visualized using the cut-off end of a P-1000 pipet tip as a speculum, and material was injected through the cervical os using a 10- $\mu$ l Hamilton syringe fitted with $0.5 \mathrm{~cm}$ of PE-10 polyethylene tubing (BD Biosciences).

In situ CFSE labeling and LPS treatment. For our basic experiments tracking DC migration from virgin uteri, $\mathrm{B} 6$ or $\mathrm{Ccr}^{-/-}$mice were given daily subcutaneous injections of $2 \mathrm{mg}$ progesterone for 3 days. On the third day, the mice were either transcervically injected with $2 \mu$ CFSE $(10 \mathrm{mM}$ in DMSO; Invitrogen) or intravenously injected with $30 \mu \mathrm{g}$ LPS (L6511; Sigma-Aldrich). The mice were sacrificed 28 hours later, a time point that was chosen because pilot experiments showed that it allowed for the optimal visualization of DC exit from the uterus and arrival in the draining LNs. At 15 hours after injection, LPS-mobilized DCs had not appreciably left the uterus nor had CFSE-labeled DCs appeared in the LNs (data not shown). The extent of CFSE labeling over the length of each uterine horn was assessed in whole mount using an Olympus MVX10 dissecting microscope equipped with GFP epifluorescence (courtesy of G. Fishell, New York University School of Medicine, New York, New York, USA).

A similar protocol was used to CFSE label and track DC migration from artificial decidua. These experiments employed B6CBAF1 females because the artificial decidual response is inconsistent in $\mathrm{B} 6$ mice. For induction of artificial decidua, sesame seed oil ( $20 \mu \mathrm{l}$; Sigma-Aldrich) was transcervically injected into pseudopregnant females 3 days after discovery of the vaginal plug. Control mice with undecidualized uteri were generated by transcervically injecting virgin females at a random stage of their estrous cycle with sesame seed oil and then giving them daily subcutaneous injections of $2 \mathrm{mg}$ progesterone from that day onward. In both cases, CFSE was transcervically injected 3 days after oil injection. To visualize LPS-induced DC mobilization during pregnancy, we intravenously injected of LPS into pregnant B6 mice that were concurrently given $2 \mathrm{mg}$ progesterone in order to compensate for the loss of ovarian function that occurs with systemic inflammation (44). Thirty nanograms of LPS was chosen for these experiments in order not to induce the rapid decidual necrosis and fetal loss seen at higher doses.

Adoptive transfers. OT-II TCR transgenic $\mathrm{CD}^{+} \mathrm{T}$ cells (45), specific for the $\mathrm{OVA}_{323-339}$ epitope presented by I-A $\mathrm{A}^{\mathrm{b}}$, were prepared, CFSE-labeled, and injected intravenously $\left(2 \times 10^{6}\right.$ cells per mouse $)$ as previously described (3).

Cell preparation. Uteri were dissected free of the mesometrium in ice-cold HBSS containing $\mathrm{Ca}^{2+}$ and $\mathrm{Mg}^{2+}$. For whole uteri and E4.5 implantation sites, the tissue was then finely minced with scissors; for later stages of gestation, the myometrium was first peeled off of the decidua using fine forceps as described previously (46), and embryos and yolk sacs were discarded. Dissected decidual tissues included increasing amounts of associated placental tissue as gestation progressed. Minced tissue was enzymatically dissociated in HBSS $/ \mathrm{Ca}^{2+} / \mathrm{Mg}^{2+}$ containing 0.28 Wunsch units (WU) Liberase Blendzyme 3 and $30 \mu \mathrm{g} / \mathrm{ml}$ DNase I (Roche) for 30 minutes at $37^{\circ} \mathrm{C}$ with intermittent trituration, washed in $\mathrm{Ca}^{2+} / \mathrm{Mg}^{2+}$-free PBS containing $5 \mathrm{mM}$ EDTA, and then incubated again in the same buffer for 15 minutes at $37^{\circ} \mathrm{C}$ prior to filtration through a $70-\mu \mathrm{m}$ cell strainer. Cells were similarly prepared from unminced uterine LNs (the paraaortic and renal LNs).

Flow cytometry. Cells were pretreated with the Fc $\gamma$ R-blocking mAb 2.4G2 and analyzed with a 3-laser 10-color BD LSRII cytometer (BD Biosciences) and FCS Express software (De Novo Software). After an initial gating on forward-versus-side scatter plots, uterine cell populations were gated on all viable leukocytes using the nucleic acid dye 7-aminoactinomycin D (7-AAD; BD Biosciences) and Pacific Orange-conjugated anti-CD45 antibodies (clone 30-F11; Invitrogen). The cells were then delineated into subsets and their activation status assessed with antibodies against MHCII (M5), Ly-6C (AL21, HK1.4), F4/80 (BM8, C1:A3-1), CD103 (2E7, M290), CD11c (HL3), CD11b (M1/70), CD86 (GL-1), purchased from BD Biosciences, BioLegend, eBioscience, or AbD Serotec and variously conjugated to Pacific Blue, FITC, PE, PE-Cy7, APC, APC-Cy7, Alexa Fluor 700, or biotin. CCR7 expression was determined by staining the cells for 30 minutes at $37^{\circ} \mathrm{C}$ with antibodies purchased from BioLegend (4B12). Visualization of adoptively transferred OT-II cells was performed as previously described (3).

Immunohistochemistry, image processing, calculation of tissue densities, and quantitative RT-PCR. These methods are described in Supplemental Methods.

In vitro migration assays. Pregnant uteri were harvested on E7.5 from B6 females mated to B6 males, and the myometrium and decidua of each implantation site were peeled away from one another as described above. For Transwell migration assays, pregnant females were injected intravenously with LPS 15 hours prior to sacrifice to stimulate the cells. After the tissues were harvested, they were enzymatically disaggregated as described above and used either as total cell suspensions or after MHCII-positive selection on a magnetic bead (MACS) column (Miltenyi Biotec). Cells $\left(10^{6}\right.$ or $\left.2 \times 10^{4}\right)$, respectively, were then applied to the top well of a $6.5-\mathrm{mm}$ diameter, 5 - $\mu \mathrm{m}$-pore-size Transwell filter (Costar 3421; Corning) in $100 \mu \mathrm{l}$ medium (RPMI containing 10\% FCS) and placed over $600 \mu \mathrm{l}$ of medium or medium plus $150 \mathrm{ng} / \mathrm{ml} \mathrm{CCL21} \mathrm{(PeproTech).} \mathrm{After} 3$ hours of incubation at $37^{\circ} \mathrm{C}$ in $5 \% \mathrm{CO}_{2}$, cells that had migrated to the bottom well were counted with a flow cytometer using a fixed collection time. Both the migrated cells and the input cells were then delineated into leukocyte subsets with the same set of antibodies used for Figure 4. The percent cell migration was determined as the number of migrated cells for a given leukocyte subset divided by the number for that subset in the input population. To determine whether DCs could emigrate from intact tissues, separated myometrial and decidual tissues were explanted at $37^{\circ} \mathrm{C}$ in $5 \% \mathrm{CO}_{2}$ for $44-46$ hours in $300 \mu \mathrm{l}$ medium with or without $150 \mathrm{ng} / \mathrm{ml} \mathrm{CCL21} \mathrm{in} \mathrm{a} \mathrm{96-well}$ plate. Cells that had emigrated into the medium were then antibody 
stained as above and counted by flow cytometry. For each mouse, the total number of migrating cells is an average of 3-5 implantation sites.

Statistics. All comparisons were performed using a 2-tailed Student's $t$ test, with a $P$ value less than 0.05 taken as statistically significant.

\section{Acknowledgments}

We thank Sergio Lira, Martin Lipp, and Reinhold Förster for providing mice; Gordon Fishell for use of a fluorescence dissecting microscope; Elisa Tagliani and Jane Skok for discussions; and Megan McCloskey, David Levy, and Nina Bhardwaj for comments on the manuscript. The NYU Histology Core Facility of the NYU
Cancer Institute provided histological support. The work was supported by a grant from the NIH (R01-AI062980).

Received for publication January 26, 2009, and accepted in revised form April 22, 2009.

Address correspondence to: Adrian Erlebacher, Department of Pathology, New York University School of Medicine, Smilow Research Center, Room 311, 550 First Avenue, New York, New York 10016, USA. Phone: (212) 263-8926; Fax: (212) 263-8211; E-mail: adrian.erlebacher@nyumc.org.
1. Trowsdale, J., and Betz, A.G. 2006. Mother's little helpers: mechanisms of maternal-fetal tolerance. Nat. Immunol. 7:241-246.

2. Moffett, A., and Loke, C. 2006. Immunology of placentation in eutherian mammals. Nat. Rev. Immunol. 6:584-594.

3. Erlebacher, A., Vencato, D., Price, K.A., Zhang, D., and Glimcher, L.H. 2007. Constraints in antigen presentation severely restrict $\mathrm{T}$ cell recognition of the allogeneic fetus. J. Clin. Invest. 117:1399-1411.

4. Kruse, A., Martens, N., Fernekorn, U., Hallmann, R., and Butcher, E.C. 2002. Alterations in the expression of homing-associated molecules at the mater$\mathrm{nal} /$ fetal interface during the course of pregnancy. Biol. Reprod. 66:333-345.

5. Dey, S.K., et al. 2004. Molecular cues to implantation. Endocr. Rev. 25:341-373.

6. Plaks, V., et al. 2008. Uterine DCs are crucial for decidua formation during embryo implantation in mice. J. Clin. Invest. 118:3954-3965.

7. Krey, G., et al. 2008. In vivo dendritic cell depletion reduces breeding efficiency, affecting implantation and early placental development in mice. J. Mol. Med. 86:999-1011.

8. Blois, S.M., et al. 2004. Lineage, maturity, and phenotype of uterine murine dendritic cells throughout gestation indicate a protective role in maintaining pregnancy. Biol. Reprod. 70:1018-1023.

9. Zarnani, A.H., Moazzeni, S.M., Shokri, F., Salehnia, M., and Jeddi-Tehrani, M. 2007. Kinetics of murine decidual dendritic cells. Reproduction. 133:275-283.

10. Behrends, J., Karsten, C.M., Wilke, S., Robke, A., and Kruse, A. 2008. Identification of ITGA4/ ITGB7 and ITGAE/ITGB7 expressing subsets of decidual dendritic-like cells within distinct microdomains of the pregnant mouse uterus. Biol. Reprod. 79:624-632.

11. Gouon-Evans, V., and Pollard, J.W. 2001. Eotaxin is required for eosinophil homing into the stroma of the pubertal and cycling uterus. Endocrinology. 142:4515-4521.

12. Pollard,J.W., Lin, E.Y., and Zhu, L. 1998. Complexity in uterine macrophage responses to cytokines in mice. Biol. Reprod. 58:1469-1475.

13. Keenihan, S.N., and Robertson, S.A. 2004. Diversity in phenotype and steroid hormone dependence in dendritic cells and macrophages in the mouse uterus. Biol. Reprod. 70:1562-1572.

14. Johansson-Lindbom, B., et al. 2005. Functional specialization of gut $\mathrm{CD} 103+$ dendritic cells in the regulation of tissue-selective $\mathrm{T}$ cell homing. J. Exp. Med. 202:1063-1073.

15. Sung, S.S., et al. 2006. A major lung CD 103 (alphaE)-beta7 integrin-positive epithelial dendritic cell population expressing Langerin and tight junction proteins. J. Immunol. 176:2161-2172.

16. Jakubzick, C., et al. 2008. Blood monocyte subsets differentially give rise to CD103+ and CD103pulmonary dendritic cell populations. J. Immunol. 180:3019-3027.
17. Sun, C.M., et al. 2007. Small intestine lamina propria dendritic cells promote de novo generation of Foxp3 T reg cells via retinoic acid. J. Exp. Med. 204:1775-1785.

18. Vermaelen, K.Y., Carro-Muino, I., Lambrecht, B.N., and Pauwels, R.A. 2001. Specific migratory dendritic cells rapidly transport antigen from the airways to the thoracic lymph nodes. J. Exp. Med. 193:51-60.

19. Forster, R., Davalos-Misslitz, A.C., and Rot, A. 2008. CCR7 and its ligands: balancing immunity and tolerance. Nat. Rev. Immunol. 8:362-371.

20. Forster, R., et al. 1999. CCR7 coordinates the primary immune response by establishing functional microenvironments in secondary lymphoid organs. Cell. 99:23-33.

21. Ohl, L., et al. 2004. CCR7 governs skin dendritic cell migration under inflammatory and steadystate conditions. Immunity. 21:279-288.

22. Ehst, B.D., Ingulli, E., and Jenkins, M.K. 2003. Development of a novel transgenic mouse for the study of interactions between CD4 and CD8 T cells during graft rejection. Am. J. Transplant. 3:1355-1362.

23. Das, S.K., Lim, H., Paria, B.C., and Dey, S.K. 1999. Cyclin D3 in the mouse uterus is associated with the decidualization process during early pregnancy. J. Mol. Endocrinol. 22:91-101.

24. Red-Horse, K., et al. 2006. Cytotrophoblast induction of arterial apoptosis and lymphangiogenesis in an in vivo model of human placentation. J. Clin. Invest. 116:2643-2652.

25. Bany, B.M., and Cross, J.C. 2006. Post-implantation mouse conceptuses produce paracrine signals that regulate the uterine endometrium undergoing decidualization. Dev. Biol. 294:445-456.

26. Roake, J.A., et al. 1995. Dendritic cell loss from nonlymphoid tissues after systemic administration of lipopolysaccharide, tumor necrosis factor, and interleukin 1. J. Exp. Med. 181:2237-2247.

27. Brandon, J.M. 1995. Macrophage distribution in decidual tissue from early implantation to the periparturient period in mice as defined by the macrophage differentiation antigens $\mathrm{F} 4 / 80$, macrosialin and the type 3 complement receptor. J. Reprod. Fertil. 103:9-16.

28. Stewart, I.J., and Mitchell, B.S. 1991. The distribution of uterine macrophages in virgin and early pregnant mice. J. Anat. 179:183-196.

29. Pollard, J.W., Hunt, J.S., Wiktor-Jedrzejczak, W., and Stanley, E.R. 1991. A pregnancy defect in the osteopetrotic (op/op) mouse demonstrates the requirement for CSF-1 in female fertility. Dev. Biol. 148:273-283.

30. Game, D.S., and Lechler, R.I. 2002. Pathways of allorecognition: implications for transplantation tolerance. Transpl. Immunol. 10:101-108.

31. Lafferty, K.J., Prowse, S.J., Simeonovic, C.J., and Warren, H.S. 1983. Immunobiology of tissue transplantation: a return to the passenger leukocyte concept. Annu. Rev. Immunol. 1:143-173.

32. Barker, C.F., and Billingham, R.E. 1968. The role of afferent lymphatics in the rejection of skin homografts. J. Exp. Med. 128:197-221.

33. Itano, A.A., et al. 2003. Distinct dendritic cell populations sequentially present antigen to CD4 T cells and stimulate different aspects of cell-mediated immunity. Immunity. 19:47-57.

34. Allenspach, E.J., Lemos, M.P., Porrett, P.M., Turka, L.A., and Laufer, T.M. 2008. Migratory and lymphoid-resident dendritic cells cooperate to efficiently prime naive CD4 T cells. Immunity. 29:795-806.

35. Farrar, J.D., and Carson, D.D. 1992. Differential temporal and spatial expression of mRNA encoding extracellular matrix components in decidua during the peri-implantation period. Biol. Reprod. 46:1095-1108.

36. Wewer, U.M., Damjanov, A., Weiss, J., Liotta, L.A., and Damjanov, I. 1986. Mouse endometrial stromal cells produce basement-membrane components. Differentiation. 32:49-58.

37. Brown, J.J., and Papaioannou, V.E. 1992. Distribution of hyaluronan in the mouse endometrium during the periimplantation period of pregnancy. Differentiation. 52:61-68.

38. Mummert, D.I., Takashima, A., Ellinger, L., and Mummert, M.E. 2003. Involvement of hyaluronan in epidermal Langerhans cell maturation and migration in vivo. J. Dermatol. Sci. 33:91-97.

39. Mummert, M.E., Mohamadzadeh, M., Mummert, D.I., Mizumoto, N., and Takashima, A. 2000. Development of a peptide inhibitor of hyaluronan-mediated leukocyte trafficking. J. Exp. Med. 192:769-779.

40. San Martin, S., Soto-Suazo, M., and Zorn, T.M. 2003. Distribution of versican and hyaluronan in the mouse uterus during decidualization. Braz.J. Med. Biol. Res. 36:1067-1071.

41. Weiss, J.M., et al. 1997. An essential role for CD44 variant isoforms in epidermal Langerhans cell and blood dendritic cell function. J. Cell Biol. 137:1137-1147.

42. Red-Horse, K. 2008. Lymphatic vessel dynamics in the uterine wall. Placenta. 29(Suppl. A):S55-S59.

43. Mitchell, M.H., Swanson, R.J., and Oehninger, S. 2002. In vivo effect of leukemia inhibitory factor (LIF) and an anti-LIF polyclonal antibody on murine embryo and fetal development following exposure at the time of transcervical blastocyst transfer. Biol. Reprod. 67:460-464.

44. Erlebacher, A., Zhang, D., Parlow, A.F., and Glimcher, L.H. 2004. Ovarian insufficiency and early pregnancy loss induced by activation of the innate immune system. J. Clin. Invest. 114:39-48.

45. Barnden, M.J., Allison, J., Heath, W.R., and Carbone, F.R. 1998. Defective TCR expression in transgenic mice constructed using cDNA-based alpha- and beta-chain genes under the control of heterologous regulatory elements. Immunol. Cell Biol. 76:34-40.

46. Nagy, A., Gertsenstein, M., Vintersten, K., and Behringer, R. 2003. Manipulating the mouse embryo: a laboratory manual. Cold Spring Harbor Laboratory Press. Cold Spring Harbor, New York, USA. 764 pp. 